\title{
Una propuesta de innovación docente para la asignatura de Comunicación Política en el Grado de Publicidad y Relaciones Públicas. Objetividad y emoción en política
}

\author{
A proposal for teaching \\ innovation for the subject of \\ Political Communication in \\ the Degree in Advertising and \\ Public Relations. Objectivity \\ and emotion in politics
}

SARA REBOllo-BuEno

ORCID: https://orcid.org/0000-0001-8179-6562

Universidad de Sevilla

Dpto. Comunicación Audiovisual y Publicidad srebollo@us.es

DOI: http://dx.doi.org/10.12795/9788447231003.062

Рp.: 1313-1332 


\section{Descripción del contexto}

La aplicación de este Ciclo de Mejora en el Aula (CIMA) se realiza a la asignatura optativa: Comunicación Política. Esta asignatura pertenece al cuarto curso del Grado en Publicidad y Relaciones Públicas de la Universidad de Sevilla. La impartición de esta materia cuatrimestral se distribuye en dos sesiones de dos horas cada una. Se trata de un grupo único formado por 80 alumnos que, debido a la crisis sanitaria de la COVID-19, acuden a clase de forma semipresencial. No obstante, las nuevas y excepcionales medidas tomadas por la Junta de Andalucía ante el aumento de casos de la COVID-19, el CIMA se aplica en sesiones totalmente telemáticas a través de la plataforma virtual.

Esta asignatura se centra en transmitir conocimientos centrados en comunicación política como, por ejemplo, la creación y uso de campañas políticas y electorales, la publicidad política, los medios de comunicación de masas, entre otros. Es relevante que el alumnado adopte conocimientos sobre el contenido ideológico de los diferentes discursos políticos, así como que desarrolle el aprendizaje de herramientas que les permitan ser capaces de analizar y formular mensajes políticos.

\section{Diseño previo del CIMA}

\section{Mapa de contenidos}

Según se puede consultar en la Figura 1, se ha dividido la materia en dos bloques: think tanks y las emociones en comunicación política. En el primer bloque, se busca hacer hincapié en la definición, las funciones, tipologías y relaciones de los medios de comunicación de masas que realizan estas instituciones, así como de las técnicas 
y estrategias de comunicación que utilizan. Se destaca la apariencia de dependencia y objetividad que tienen estas instituciones. De esta forma, se pone en comparación con el segundo bloque, en el cual se muestra que las emociones juegan un papel fundamental en las campañas y actuaciones de comunicación política, sobre todo, se destaca la emoción del miedo y la esperanza. Gracias a esta organización del contenido, se puede observar el "juego" político entre el uso de lo objetivo/racional y lo emocional que se desempeña en comunicación política. Estos conocimientos son esenciales, no solo para el mundo laboral al que se enfrentarán los alumnos, sino para entender el contexto político y social.

Al ser dos bloques, el CIMA parte de dos problemas $(P)$ :

P1: ¿Usan los medios de comunicación de masas datos objetivos sobre posturas y cuestiones politicas?

P2: En comunicación política, ¿qué se usa más la racionalidad o la emocionalidad?

Teniendo como punto de partida estos dos problemas, la sesión irá desarrollándose en torno a dichas cuestiones, con el fin de ir desglosando la materia e irla impartiendo con la interacción de los alumnos, los cuales sabrán en todo momento qué queremos aprender con la sesión.

Como se sigue observando en la Figura 1, el CIMA recoge tres tipos de contenidos: conceptuales, procedimentales y actitudinales. Los conceptuales se centran en aquellas cuestiones más teóricas que son esenciales para comprender los fenómenos comunicativos, como, por ejemplo, los think tanks y sus funciones. Los procedimentales buscan solventar cuestiones más prácticas, la aplicabilidad. Por último, los actitudinales pretenden desarrollar competencias en el alumnado más centradas en lo ético y crítico. Estos últimos se condensan al final de los bloques, pues se espera que el alumnado ya haya adquirido tanto los conceptuales como procedimentales para poder generar un debate crítico en el aula. 


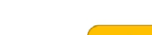

Dersectiva de indenendencialkin ánima de lirect

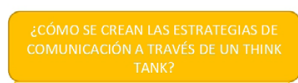

Jornadas, conferencias, etc. Reuniones con los medios. Publicación de libros, artículos.

etc.
Entre otros.

$\longrightarrow$ Y... ¿a los otros?
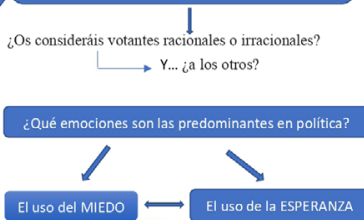

- Creación del enemigo -Estudio del contexto

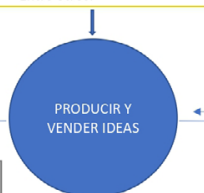

Rol laboral (perspectiva citica)

Figura 1. Mapa de contenidos

\section{Modelo metodológico posible}

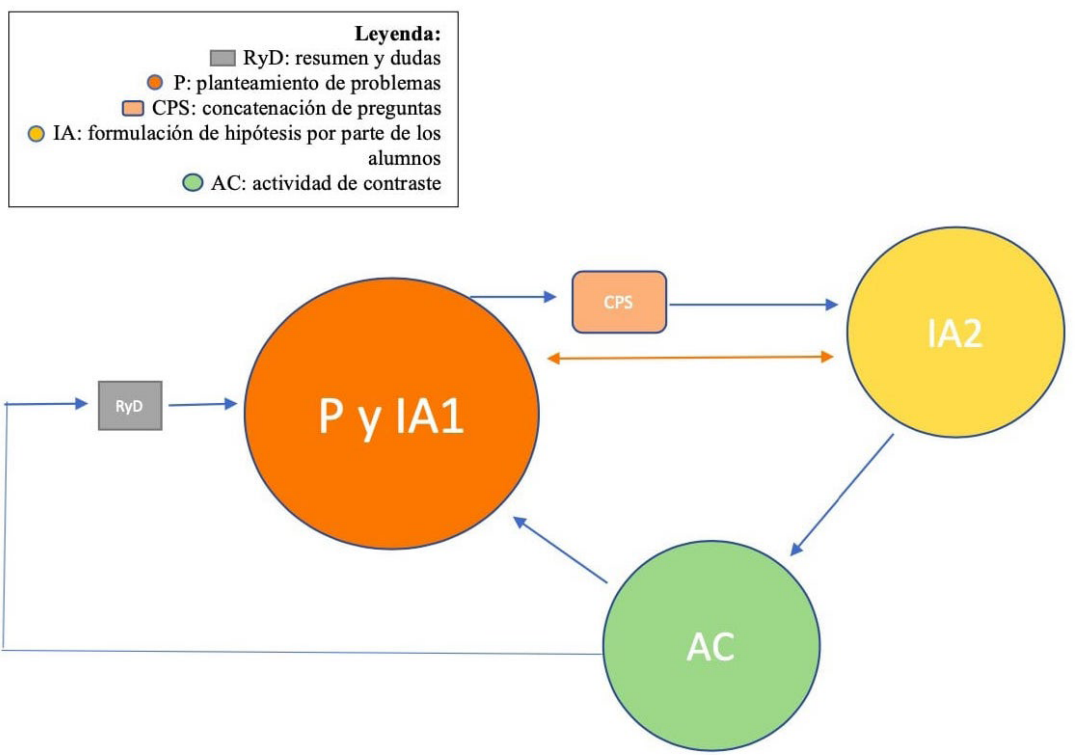

Figura 2. Modelo metodológico posible

Ciclos de Mejora en el Aula (2020). Experiencias de Innovación Docente de la US (c) (i) $\odot$ Esta obra se distribuye con la licencia Creative Commons 
El modelo metodológico presentado se concreta en el diseño de una secuencia de actividades. Dicha secuencia desglosa detalladamente la temporalización, la explicación y la tipología de cada una de las actividades que se realizan durante el CIMA.

Tabla 1. Secuencia de actividades sesión 1

\begin{tabular}{|c|c|c|c|}
\hline \multicolumn{4}{|c|}{ Sesión 1 (dos horas) } \\
\hline Act. 1 & PLANTEAMIENTO DEL PROBLEMA CLAVE & 30min. & P y IA \\
\hline \multicolumn{4}{|c|}{$\begin{array}{l}\text { Descripción de la actividad: } \\
\text { La clase se inicia planteando en la diapositiva la pregunta: ¿Usan los medios } \\
\text { de comunicación de masas datos objetivos sobre posturas y cuestiones } \\
\text { políticas? } \\
\text { Se instará a los estudiantes a que den su opinión y las argumenten usando } \\
\text { grupos de ocho personas. Una vez que pasen unos } 10 \text { min., se preguntará a } \\
\text { qué conclusiones han llegado, utilizando un portavoz por grupo para que se } \\
\text { ponga en común las ideas principales. } \\
\text { Una vez se genere cierto consenso, se plantea la pregunta secundaria: } \\
\text { ¿Cualquier centro de investigación aporta datos objetivos y fiables? Para } \\
\text { resolver esta pregunta, se utilizará el mismo método de grupos y portavoces. } \\
\text { La docente solo intervendrá con preguntas dependiendo de las respuestas } \\
\text { de los alumnos a dicha cuestión. De momento, no hay respuestas por su } \\
\text { parte. }\end{array}$} \\
\hline \multicolumn{4}{|c|}{$\begin{array}{l}\text { Contenido: } \\
\text { Preparación para presentar el concepto de think tank. }\end{array}$} \\
\hline Act. 2 & EN BUSCA DE... ¿THINK TANKS? & 40min. & AC \\
\hline \multicolumn{4}{|c|}{$\begin{array}{l}\text { Descripción de la actividad: } \\
\text { Una vez cerrado este primer pequeño debate, se insta a los alumnos a que } \\
\text { utilicen sus ordenadores o móviles para buscar en internet páginas webs de } \\
\text { think tanks españoles y que, por favor, recopilen las capturas de pantalla de } \\
\text { la parte de estas webs tituladas: "Quiénes somos" o de presentación. Se les } \\
\text { pedirá que las hagan llegar a mi correo o al Drive de la clase. } \\
\text { Una vez tenga en mi poder todas las búsquedas de los alumnos, empiezo } \\
\text { a abrirlas en la pantalla para que todos vean todas y les voy pidiendo que } \\
\text { me vayan diciendo dos cosas: cuáles son las partes de las definiciones que } \\
\text { se van repitiendo y, además, qué consideran que es clave para entender las } \\
\text { funciones de esas instituciones. }\end{array}$} \\
\hline
\end{tabular}

Ciclos de Mejora en el Aula (2020). Experiencias de Innovación Docente de la US Esta obra se distribuye con la licencia Creative Commons Reconocimiento-NoComercial-SinObraDerivada $\quad 4.0$ Internacional (CC BY-NC-ND 4.0.) 
Una vez tengamos todas las partes apuntadas en la pizarra, les expongo la definición de Vargas (2012) para que puedan comparar. Les preguntaré qué diferencias ven entre lo que los think tanks dicen que son y lo que los teóricos afirman que son.

Contenido:

Construcción del concepto de think tanks y comenzamos a vislumbrar las funciones de estas instituciones. Asi como conocer think tanks españoles y la importancia de la dependencia privada y pública de este tipo de instituciones.

\begin{tabular}{|c|c|c|c|}
\hline Act. 3 & LABORATORIOS DE IDEAS & $15 \mathrm{~min}$. & AC \\
\hline \multicolumn{4}{|c|}{$\begin{array}{l}\text { Descripción de la actividad: } \\
\text { Se les pide a los alumnos que ahora, sabiendo ese sesgo existente en las } \\
\text { webs que han visitado, que revisen esas mismas webs y digan cuál es el } \\
\text { objetivo de esas instituciones. }\end{array}$} \\
\hline \multicolumn{4}{|c|}{$\begin{array}{l}\text { Contenidos: } \\
\text { Think tanks como laboratorios de ideas, objetivo: producir y vender ideas. }\end{array}$} \\
\hline Act. 4 & LOBBY VS. THINK TANKS & $5 \mathrm{~min}$. & AC \\
\hline \multirow{2}{*}{\multicolumn{4}{|c|}{$\begin{array}{l}\text { Descripción de la actividad: } \\
\text { Leves apuntes teóricos sobre las diferencias entre lobby y think tanks } \\
\text { (no tiene mucha importancia, pero si no se menciona puede que genere } \\
\text { confusión con otras asignaturas). } \\
\text { Contenidos: } \\
\text { Delimitación conceptual de fenómenos parecidos. }\end{array}$}} \\
\hline & & & \\
\hline Act. 5 & RESUMEN Y DUDAS & & \\
\hline \multicolumn{4}{|c|}{$\begin{array}{l}\text { Descripción de la actividad: } \\
\text { Se ofrece un pequeño resumen sobre lo que hemos visto en la sesión y se } \\
\text { pregunta si han surgido dudas. }\end{array}$} \\
\hline \multicolumn{4}{|c|}{$\begin{array}{l}\text { Se deja unos } 10-15 \text { min. de margen para evitar la falta de tiempo, por si } \\
\text { algún debate se extiende. }\end{array}$} \\
\hline
\end{tabular}

Tabla 2. Secuencias de actividades sesión 2

\begin{tabular}{|l|l|c|c|}
\hline \multicolumn{4}{|c|}{ Sesión 2 (dos horas) } \\
\hline Act. $\mathbf{1}$ & \multicolumn{1}{|c|}{ RESUMEN Y DUDAS } & \multicolumn{2}{|c|}{ 5min. } \\
\hline $\begin{array}{l}\text { Descripción de la actividad: } \\
\text { La clase comienza haciendo un pequeño resumen sobre lo visto en la sesión } \\
\text { del lunes pasado y, además, preguntando si existen dudas. }\end{array}$ \\
\hline Act. $\mathbf{2}$ & PLANTEAMIENTO DEL PROBLEMA & $\mathbf{1 5 m i n . ~}$ & IA \\
\hline
\end{tabular}

Ciclos de Mejora en el Aula (2020). Experiencias de Innovación Docente de la US Esta obra se distribuye con la licencia Creative Commons Reconocimiento-NoComercial-SinObraDerivada $\quad 4.0$ Internacional (CC BY-NC-ND 4.0.) 
Descripción de la actividad:

La clase se inicia planteando en la diapositiva la pregunta: ¿Cómo se relacionan estas instituciones con los medios?

Se instará a los estudiantes a que den su opinión y las argumenten usando grupos de 8 personas. Una vez que pasen unos 10 min., se preguntará a qué conclusiones han llegado, utilizando un portavoz por grupo para que se ponga en común las ideas principales.

Contenidos:

Relación entre los medios de comunicación de masas y los think tanks.

\begin{tabular}{|c|c|c|c|}
\hline Act.3 & IMAGINEMOS UN POCO & $40 \mathrm{~min}$. & AC \\
\hline
\end{tabular}

Descripción de la actividad:

Se plantea el siguiente problema en la diapositiva para que lo resuelvan en grupos de 5-8 personas:

Imaginemos que trabajamos para FAES (un think tank español) y tenemos un nuevo estudio acerca del impacto económico en las clases altas, medias y bajas españolas tras la crisis de la COVID-19. Hemos extraído unas conclusiones relevantes. Podríais explicarnos qué factores son útiles para generar interés en los medios y conseguir que publiquen nuestras conclusiones. Indica 4 factores a tener en cuenta.

Una vez hayan acabado, se pondrán en común y se les facilitará las funciones expuestas por los manuales de teoría sobre estas instituciones.

Contenidos:

Funciones y relaciones de los think tanks con los medios de comunicación de masas.

Destacando la importancia de la apariencia de independencia, así como conocer más profundamente uno de los principales think tanks españoles: FAES.

\begin{tabular}{|l|l|l|l|}
\hline Act. 4 & SIGAMOS IMAGINANDO & $35 \mathrm{~min}$. & AC \\
\hline
\end{tabular}

Descripción de la actividad:

Siguiendo con los grupos y el supuesto de la actividad 3 (pertenecemos a la plantilla de FAES). Se les propone a los alumnos que piensen ahora cómo se difunden investigaciones científicas para que sean acogidas por el interés de sus públicos, desde los medios de comunicación de masas hasta la audiencia masiva. En definitiva, que piensen acerca de qué estrategias de comunicación llevarían acabo para este supuesto.

Se pone en común todo lo aportado y se les aportará más ejemplos sobre estrategias, técnicas y tácticas que usen este tipo de instituciones.

Contenidos:

Estrategias, técnicas y tácticas de comunicación de los think tanks en base a las relaciones con sus públicos.

\begin{tabular}{l|l|l} 
Act. 5 & RESUMEN Y DUDAS & $5 \mathrm{~min}$. \\
\hline
\end{tabular}

Ciclos de Mejora en el Aula (2020). Experiencias de Innovación Docente de la US Esta obra se distribuye con la licencia Creative Commons Reconocimiento-NoComercial-SinObraDerivada $\quad 4.0$ Internacional (CC BY-NC-ND 4.0.) 
Descripción de la actividad:

Conociendo ya lo que son y cómo actúan este tipo de instituciones, se les pregunta acerca de su perspectiva ética sobre estas estrategias de comunicación.

\begin{tabular}{|l|l|l|l|}
\hline Act. 6 & DEBATE FINAL/PERSPECTIVA CRITICA & 5-10min. & AC \\
\hline
\end{tabular}

Descripción de la actividad:

Conociendo lo que son y cómo actúan este tipo de instituciones, se les pregunta acerca de su perspectiva ética sobre estas estrategias de comunicación y el uso de las emociones en política.

Contenido:

Perspectiva crítica acerca de estas instituciones y visión del rol laboral.

Se deja unos 10-15min. de margen para evitar la falta de tiempo, por si algún debate se extiende.

Tabla 3. Secuencias de actividades sesión 3

\begin{tabular}{|c|c|c|c|}
\hline \multicolumn{4}{|c|}{ Sesión 3 (dos horas) } \\
\hline Act.1 & RESUMEN Y DUDAS & \multicolumn{2}{|c|}{ 5-10min. } \\
\hline \multicolumn{4}{|c|}{$\begin{array}{l}\text { Descripción de la actividad: } \\
\text { La clase comienza haciendo un pequeño resumen sobre lo visto en la sesión } \\
\text { del jueves pasado y, además, preguntando si existen dudas. }\end{array}$} \\
\hline Act.2 & PLANTEAMIENTO DEL PROBLEMA CLAVE & 35min. & IA y AC \\
\hline \multicolumn{4}{|c|}{$\begin{array}{l}\text { Descripción de la actividad: } \\
\text { La clase se inicia planteando en la diapositiva la pregunta: En comunicación } \\
\text { política, ¿qué se usa más la racionalidad o la emocionalidad? Se instará a los } \\
\text { estudiantes a que den su opinión y las argumenten. Una vez se genere cierto } \\
\text { consenso, se plantea la pregunta secundaria: ¿Os consideráis a vosotros } \\
\text { mismos votantes racionales e irracionales? } \\
\text { Para más tarde preguntar: ¿Y al resto de personas? } \\
\text { Una vez hayan dado sus argumentos y partiendo de estos mismos, se les } \\
\text { explicará la diferencia entre racionalidad y racionalización. Y, por supuesto, } \\
\text { cómo los votantes (y todos) se sitúan en esta última. }\end{array}$} \\
\hline \multicolumn{4}{|c|}{$\begin{array}{l}\text { Contenidos: } \\
\text { Teoría sobre la no racionalidad de los votantes y diferencia entre racionalidad } \\
\text { y racionalización. }\end{array}$} \\
\hline Act.3 & IMAGINEMOS UN POCO & 1h & IA y AC \\
\hline
\end{tabular}

Ciclos de Mejora en el Aula (2020). Experiencias de Innovación Docente de la US Esta obra se distribuye con la licencia Creative Commons Reconocimiento-NoComercial-SinObraDerivada $\quad 4.0$ Internacional (CC BY-NC-ND 4.0.) 
Descripción de la actividad:

Sabiendo que el uso de las emociones es mayoritario gracias a la racionalización y no la racionalidad, se les plantea a los estudiantes la pregunta: ¿Cuáles son las emociones predominantes en política?

Para que la puedan solucionar, se les insta a una nueva actividad que se proyectará en las diapositivas. Dice así:

Imaginemos que somos jefes de campaña y tenemos que prepararnos para unas elecciones tras la COVID-19. Obviamente, es un contexto dado a la emocionalidad por todo lo que ha implicado para el país. Para esto, tienen que buscar en campañas anteriores del ciclo electoral de 2019 qué emociones han utilizado sus partidos y decidir si mantienen la misma estrategia de comunicación con esa emoción (o emociones, puede haber más de una) o si, por el contrario, cambian su decisión en base a lo anterior.

Una vez hayan finalizado su búsqueda y hayan tenido que tomar esas dos decisiones por grupo: saber qué emoción usaban en el ciclo electoral de 2019 y decidir con qué seguir a nivel estratégico en el nuevo contexto, se pondrán en común sus resultados.

Contenidos:

Uso de las emociones en política.

Uso de las emociones en las campañas electorales españolas (diferenciando entre partidos).

Se les irá ayudando grupo por grupo también para poder enfocar la actividad a la práctica final. Así se irán resolviendo dudas y, además, ayudándoles a generar decisiones estratégicas de cara a la práctica (suelen temerle mucho por la cantidad de trabajo).

\begin{tabular}{|c|c|c|}
\hline Act.4 & RESUMEN Y DUDAS & 5-10min. \\
\hline \multicolumn{3}{|c|}{$\begin{array}{l}\text { Descripción de la actividad: } \\
\text { Se ofrece un pequeño resumen sobre lo que hemos visto en la sesión y } \\
\text { se pregunta si han surgido dudas. Asimismo, se les pedirá que, habiendo } \\
\text { participado en esta actividad, qué conclusiones sacan al respecto para el } \\
\text { próximo día comentarlas. }\end{array}$} \\
\hline
\end{tabular}

Se deja unos 10-15min. de margen para evitar la falta de tiempo, por si algún debate se extiende.

Tabla 4. Secuencias de actividades sesión 4

\begin{tabular}{|l|l|l|}
\hline \multicolumn{2}{|l|}{ Sesión $\mathbf{4}$ (dos horas) } \\
\hline Act.1 & RESUMEN Y DUDAS & 5-10min \\
\hline Descripción de la actividad: \\
La clase comienza haciendo un pequeño resumen sobre lo visto en la \\
sesión del lunes pasado y, además, preguntando si existen dudas. Así \\
como afirmando que esa será mi última o penúltima clase (dependiendo \\
de una cuestión del otro profesor)
\end{tabular}

Ciclos de Mejora en el Aula (2020). Experiencias de Innovación Docente de la US Esta obra se distribuye con la licencia Creative Commons Reconocimiento-NoComercial-SinObraDerivada $\quad 4.0$ Internacional (CC BY-NC-ND 4.0.) 


\begin{tabular}{l|l|c|c|}
\hline Act.2 & \multicolumn{1}{|c|}{ PLANTEAMIENTO DEL PROBLEMA } & 25min. & Al \\
\hline Descripción de la actividad: \\
En el resumen anterior, recupero la actividad de la sesión pasada y les pido \\
que, haciendo un esfuerzo de memoria, me respondan a la pregunta que lo \\
empezó todo: ¿Cuáles son las emociones predominantes en política? \\
Como he analizado previamente las campañas, sé que las emociones \\
predominantes que saldrán será el miedo, la esperanza y la ira. Se les \\
explicará que tienen en común que son emociones básicas y se les pedirá \\
que den su opinión respecto a su uso. \\
Contenido: \\
El uso del miedo y la esperanza en comunicación política.
\end{tabular}

\begin{tabular}{|c|c|c|c|}
\hline Act.3 & DESMENUZAR PARA VOLVER A CONSTRUIR & $40 \mathrm{~min}$. & AC \\
\hline
\end{tabular}

Descripción de la actividad:

Una vez se haya finalizado el debate anterior, se les pide que busquen una campaña de comunicación política, o utilicen la que analizaron en la sesión anterior, y desglosen los argumentos para poder ver los factores que ayudan a utilizar el miedo (en los mismos grupos de la sesión anterior).

Una vez finalizada la búsqueda y la identificación, de nuevo, lo ponemos en común. La docente irá apuntando los factores en la pizarra según van diciéndoselo los alumnos.

Una vez finalizado, se expondrá en la diapositiva las técnicas dadas por los manuales y teóricos y se pondrán en comparación con las que ellos han encontrado y se explicarán.

Contenidos:

Las técnicas para el uso del miedo en comunicación política y enlace con el uso de la esperanza.

\begin{tabular}{|c|c|c|c|}
\hline Act.4 & EL JUEGO DEL MIEDO Y LA ESPERANZA & $15 \mathrm{~min}$. & AC \\
\hline
\end{tabular}

Descripción de la actividad:

El miedo y la esperanza son esenciales en comunicación política: si generas miedo sin aportar una esperanza probablemente no sea eficaz. Por tanto, se les plantea a los alumnos un listado en las diapositivas de argumentos generadores de miedo usados en política (ej: esas políticas económicas llevarán a la destrucción del Estado de Bienestar o los inmigrantes vienen a quitarnos el trabajo). Y "jugamos" entre todos, a terminar el argumento político usando la esperanza.

Contenidos:

Uso del miedo y la esperanza en comunicación política.

Ejemplos de campañas reales.

\begin{tabular}{|c|c|c|c|}
\hline Act.5 & DEBATE FINAL/PERSPECTIVA CRITICA & $15 \mathrm{~min}$. & Al \\
\hline
\end{tabular}

Ciclos de Mejora en el Aula (2020). Experiencias de Innovación Docente de la US Esta obra se distribuye con la licencia Creative Commons Reconocimiento-NoComercial-SinObraDerivada $\quad 4.0$ Internacional (CC BY-NC-ND 4.0.) 
Descripción de la actividad:

Una vez entendido el proceso y las tácticas comunicativas que se utilizan, se plantea que: Conociendo lo dado la semana pasada (esfuerzos políticos por aparentar objetividad), y que las emociones son más usadas en política que los argumentos racionales: ¿cuál de las dos es más importante: lo racional o lo emocional?

Contenidos:

Pensamiento crítico sobre el uso de las emociones en comunicación política (rol laboral).

\begin{tabular}{|c|c|c|}
\hline Act. 6 & RESUMEN Y DUDAS & $10-15 \mathrm{~min}$ \\
\hline \multicolumn{3}{|c|}{$\begin{array}{l}\text { Descripción de la actividad: } \\
\text { Se ofrece un pequeño resumen sobre lo que hemos visto en la sesión y se } \\
\text { pregunta si han surgido dudas. O si tienen alguna de cara a la práctica, pues } \\
\text { puede que no les vea más hasta ese día. }\end{array}$} \\
\hline
\end{tabular}

\section{Cuestionario inicial-final}

Para poder valorar el punto de partida de los estudiantes, conociendo así qué dominio tienen de la materia que se les va a impartir, se ha repartido un cuestionario inicial de cuatro preguntas correspondientes a las temáticas a tratar en las sesiones.

1. ¿Qué predomina más en comunicación política: los argumentos racionales o los emocionales? Justifica brevemente tu respuesta.

2. ¿Son los votantes racionales a la hora de ejercer su derecho al voto o su afiliación a un partido? Justifica brevemente tu respuesta.

3. En comunicación política: ¿las campañas se centran en certezas científicas para apoyar sus argumentos? Justifica brevemente tu respuesta

4. ¿Piensas que existen unas instrucciones o pasos a seguir para usar las emociones en comunicación política? ¿Cuáles?

Ciclos de Mejora en el Aula (2020). Experiencias de Innovación Docente de la US Esta obra se distribuye con la licencia Creative Commons 


\section{Aplicación del CIMA}

\section{Diario de sesiones}

La primera sesión comienza con poca intervención. No obstante, la primera actividad de contraste genera mucha participación e interés por parte de los alumnos, pues la gran mayoría participa e intentan intervenir a través de sus portavoces. De esto se destaca el interés en la búsqueda con dispositivos electrónicos. Cuando se les ha presentado la definición teórica, ellos mismos han ido señalando las diferencias y preguntando por qué existía ese desnivel entre lo que las propias organizaciones dicen que son y lo que realmente son. Esto ha creado un debate en clase que ha facilitado el paso a la siguiente actividad. Esta tiene menos participación, probablemente por cansancio o saturación, por lo que la profesora tuvo que alentar a la participación. Para finalizar la clase, se solucionan algunas dudas y se realiza un pequeño resumen.

La segunda sesión destaca porque, desde el inicio, existió interacción. Esto puede deberse a que se adentran en una materia que les interesa más: los medios de comunicación. La actividad de contraste conllevó más tiempo del esperado pues se dio por hecho que conocían qué era la FAES pero, al no ser así, tuvieron que dedicarse unos diez minutos a explicar de qué se trataba exactamente. Esta actividad aumentó la motivación y muchos participaron. Al ver que sus respuestas coincidían con las de los manuales, se generaba satisfacción en ellos e interactuaban entre ellos. Al igual que en la primera, la siguiente actividad de contraste provoca un descenso de la participación y, por ende, de la atención. Sin embargo, el debate con perspectiva crítica que se plantea al final de la sesión provoca una vuelta a la implicación de los alumnos en la clase, llegando a conclusiones de gran interés. De hecho, tuvo que cortarse, pues el tiempo de la sesión se agotaba 
En la tercera, parece que hay un ambiente cansado y apático, provocando que la pregunta planteada lleve a la unanimidad desde el principio por lo que la puesta en común es prácticamente inexistente. No obstante, la actividad de las emociones consigue que los alumnos participen y presentan soluciones muy interesantes y cuidadas estratégicamente, llegando a defenderlas ante sus compañeros, consiguiendo así generar un diálogo. En esta sesión hay que detenerse para explicar más profundamente la diferencia entre algunos conceptos que parecen no haber quedado claros. Se cierra la sesión haciendo un resumen de lo expuesto en clase.

La última sesión, a pesar de estar más cansados, se consiguen obtener buenas conclusiones sobre las actividades que se les plantean en la clase y el contexto político, enfocándolo a la actualidad social, económica y política. La actividad cuatro de esta sesión genera mucha participación, pues se hace en grupo único y lo ven como un "juego". Sin embargo, captan perfectamente el uso entre el miedo y la esperanza en los discursos y campaña políticas, gracias a poder analizar diferentes ejemplos. En el debate final no entran con tanta intensidad como la semana anterior, pero da para que debatan unos 15-20 minutos. Más tarde, resuelvo un par de dudas y hago un pequeño resumen. Con esto, la clase se termina.

\section{Escaleras de aprendizaje}

Gracias al cuestionario anteriormente presentado, se ha podido desarrollar una herramienta de evaluación: las escaleras de aprendizaje. Esta herramienta ha sido útil tanto para ver el punto de partida de los alumnos, tal y como se mencionó anteriormente, como para poder ver la evolución de los mismos. De hecho, se puede observar perfectamente en las siguientes páginas el proceso de aprendizaje.

Ciclos de Mejora en el Aula (2020). Experiencias de Innovación Docente de la US Esta obra se distribuye con la licencia Creative Commons 
Pregunta 1: ¿Qué predomina más en comunicación política: los argumentos racionales o los emocionales? Justifica brevemente tu respuesta.

Conocimiento de la formación de discursos políticos basados en lo emocional y racional (argumentación política).

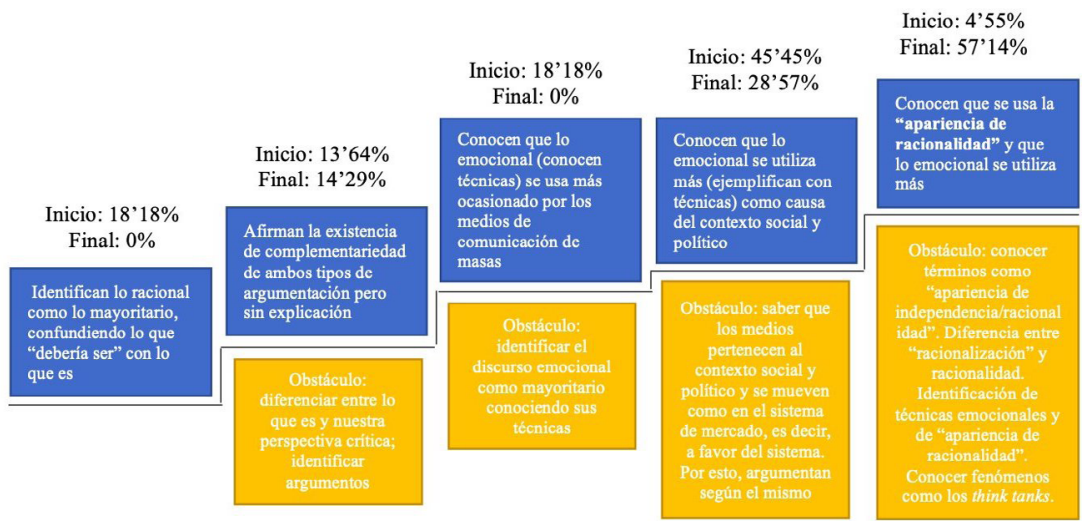

Figura 3. Escalera de aprendizaje pregunta 1

En esta primera escalera, se observa que, en general, los alumnos han comprendido la diferencia entre objetividad y apariencia de objetividad. De hecho, como muestra el porcentaje final, la mayoría ya saben que ambos bloques: lo emocional y la apariencia de objetividad, es igual de importante y son componentes esenciales para el discurso político y las estrategias de comunicación política.

Pregunta 2: ¿Son los votantes racionales a la hora de ejercer su derecho al voto o su afiliación a un partido? Justifica brevemente tu respuesta.

Conocimiento sobre el receptor (votante) a la hora de recibir discursos políticos y argumentación política, así como conocer qué genera más atención en ellos.

Ciclos de Mejora en el Aula (2020). Experiencias de Innovación Docente de la US Esta obra se distribuye con la licencia Creative Commons 


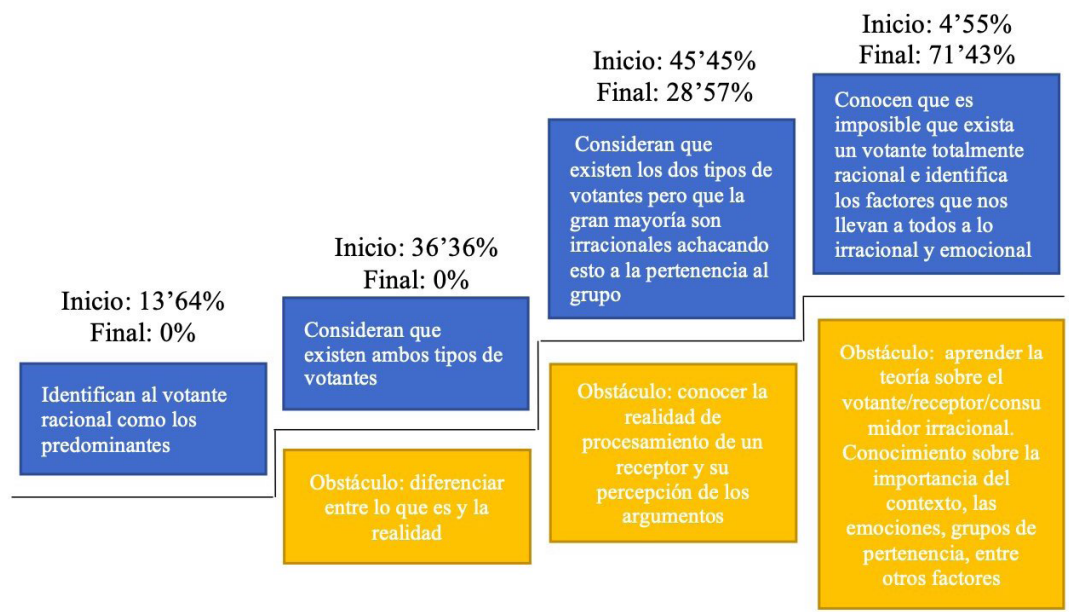

Figura 4. Escalera de aprendizaje pregunta 2

El mayor avance se ha dado en la segunda pregunta donde parece que la gran mayoría ha entendido que no existe un votante totalmente racional. Esto puede relacionarse con el debate que se desarrolló en clase, donde los propios alumnos se dieron cuenta de que consideraban al resto como votantes irracionales y a ellos mismos como racionales. De hecho, se pudieron explicar que todos estamos bajo condiciones sociales, políticas, económicas, culturales, etc. que imposibilitan la racionalidad absoluta, profundizando así la teoría del votante irracional.

Pregunta 3: En comunicación política, ¿las campañas se centran en certezas cientificas para apoyar sus argumentos? Justifica brevemente tu respuesta.

Conocimiento sobre la existencia y el uso por parte de los partidos políticos de las técnicas de "apariencia" de objetividad a través de instituciones que se autodefinen como científicas, como los think tanks.

Ciclos de Mejora en el Aula (2020). Experiencias de Innovación Docente de la US Esta obra se distribuye con la licencia Creative Commons 


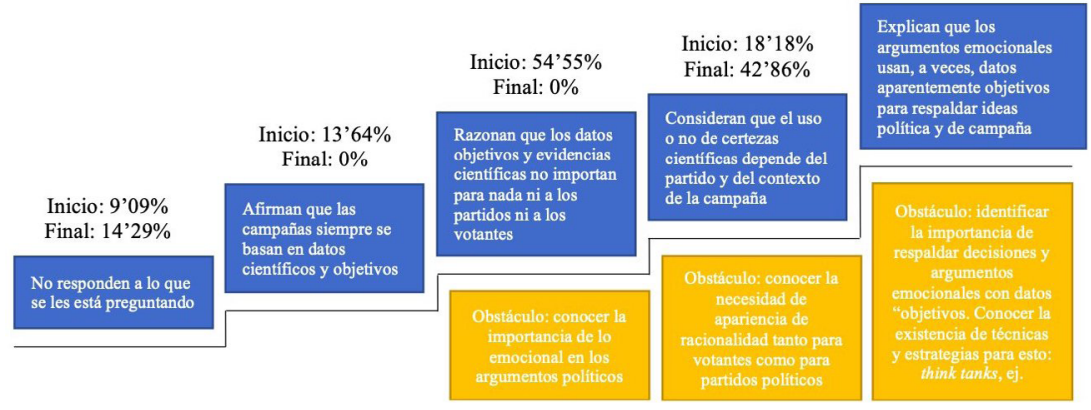

Figura 5. Escalera de aprendizaje pregunta 3

Gracias a la escalera 3, se descubre que, aunque los alumnos han entendido el concepto de "apariencia de objetividad" y su aplicabilidad en campañas y estrategias de comunicación política, siguen sin considerarlos esenciales para respaldar lo emocional. La otra mitad parece que sí ha comprendido este juego estratégico. A lo mejor, en vez de actividad de contraste, hubiese sido mejor una Al (actividad de formulación de hipótesis).

Pregunta 4: ¿Piensas que existen unas instrucciones o pasos a seguir para usar las emociones en comunicación política? ¿Cuáles? Indica 3.

Conocimiento sobre las técnicas y tácticas de comunicación para el uso de emociones en política.

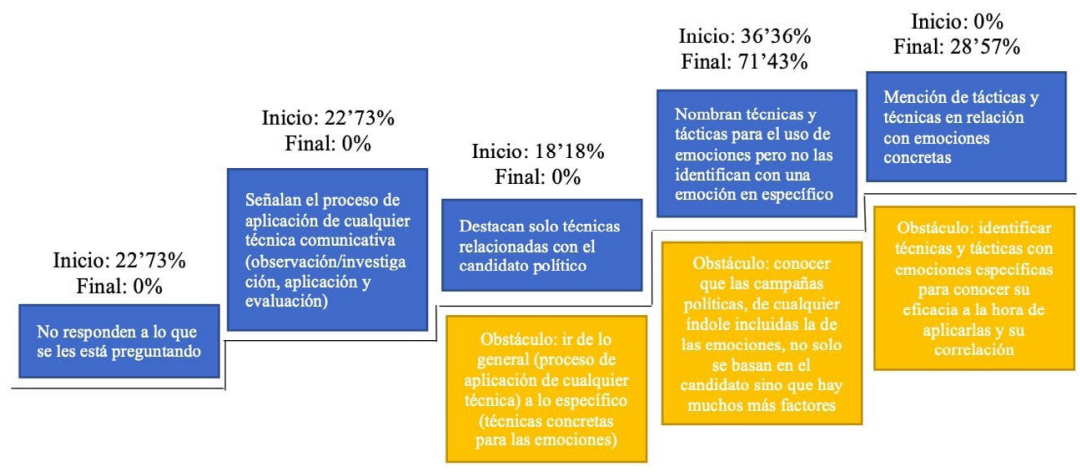

Figura 6. Escalera de aprendizaje pregunta 4

Ciclos de Mejora en el Aula (2020). Experiencias de Innovación Docente de la US Esta obra se distribuye con la licencia Creative Commons Reconocimiento-NoComercial-SinObraDerivada Internacional (CC BY-NC-ND 4.0.) 
Por último, los alumnos han retenido estrategias y técnicas del uso de las emociones en comunicación políticas, pero parece que hay cierta dificultad para poder relacionarlas con las emociones que se buscan generar. Puede que hubiese sido más adecuado realizar en la clase, entre todos, algún tipo de cuadro o esquema para que lo tuvieran visualmente delante.

\section{Evaluación del CIMA}

Atendiendo a todo lo transcurrido en estas semanas, tanto al aprendizaje propio como al resultado final referenciado en la evolución de los alumnos, queda patente el éxito de la aplicación del Ciclo de Mejora. Gracias al CIMA, se ha dejado patente la importancia del papel activo del alumno en el proceso de aprendizaje, dejando atrás las clases magistrales que poco, o nada, tenían que ver con la verdadera asimilación y procesamiento de la materia. De hecho, en el presente CIMA se han tenido en cuenta dos ejes que se han considerado vertebrales. En primer lugar, la interacción de con los alumnos, pues es un pilar esencial, no solo para poder saber cómo funciona la clase y conocer si están entendiendo y asimilando los conocimientos, sino, también, con el objetivo de evolucionar tanto a nivel individual como grupal. En segundo lugar, el otro pilar esencial del presente modelo didáctico, es el desarrollo del pensamiento crítico en el alumnado. Gran parte del aprendizaje es conseguir que usen la perspectiva crítica, consiguiendo emprender ideas nuevas, visiones diferentes y puedan ir más allá evitando, lo máximo posible, las tautologías.

Respecto a los contenidos se destaca el éxito de enseñar a través de problemas/preguntas, así como la importancia de dividir los diferentes tipos de contenidos y conocerlos, con el objetivo de interrelacionarlos y saber cómo enseñar cada uno de ellos. De esta forma, el contenido es abordado por los estudiantes desde diferentes 
ópticas pudiendo desarrollar en ellos distintas herramientas que les serán útiles en un futuro. El modelo metodológico presentado consigue que los alumnos trabajen de forma triangular: problemas/preguntas, actividades de formulación de hipótesis y actividades de contraste. En las que siempre exponen sus ideas primero para, más tarde, contrastar lo que ellos han concluido con el temario y poder ponerlo en común. Gracias a este tipo de metodología los debates en el aula son muy comunes, provocando que la implicación del alumnado aumente.

Por último, se destaca la importancia de la evaluación, pues se ha podido detectar que varios de los obstáculos de los alumnos, como puede observarse en las escaleras, se deben al corte crítico. Con corte "crítico" me refiero a que, a veces, equivocan lo que "debería hacerse" con lo que "se hace", entonces sus respuestas y análisis se escudan en su percepción crítica, sesgándola. Asimismo, se ha dejado en evidencia la importancia de entender en qué punto de partida están los alumnos para poder llegar a un punto "final", es decir, a dónde queremos llevarlos dentro de la asignatura. Por ejemplo, gracias a la evaluación, se han obtenido resultados tan importantes a considerar como que las actividades de contrastes soy muy útiles cuando se trata de enseñar conceptos, es decir, definiciones de fenómenos comunicativos. En cambio, si se trata de mostrar estrategias y técnicas de dichos fenómenos, parece mucho más eficaz el uso de actividades de formulación de hipótesis, donde ellos mismos puedan debatir y sacar conclusiones acerca de las diferentes técnicas, tácticas y estrategias. Por tanto, la evaluación es esencial para poder construir, no solo el CIMA en el que se esté trabajado, sino también para construir y sacar conclusiones contundentes para próximas sesiones.

Ciclos de Mejora en el Aula (2020). Experiencias de Innovación Docente de la US Esta obra se distribuye con la licencia Creative Commons 


\section{Principios Didácticos Personales}

De la evaluación comentada con anterioridad se desglosan los siguientes principios didácticas:

1. La interacción como base esencial para la construcción de una docencia que abogue por el aprendizaje real de los estudiantes.

2. El desarrollo del pensamiento crítico del alumnado como pilar básico.

3. El uso de una metodología que abogue por partir de problemas/preguntas para que los alumnos tengan siempre claro qué queremos resolver (aprender) y por qué.

4. Aplicar una metodología que sepa agrupar actividades de diferente índole consiguiendo así mantener el interés del alumnado.

5. Debates de perspectiva crítica que permita a los estudiantes expresarse y poner en común ideas esenciales para el futuro de su rol laboral y de la sociedad en general.

6. Realizar una evaluación de la docencia impartida, con el fin de pulir y construir Ciclos de Mejora que terminen por convertirse en propuestas docentes cada vez más exigentes en términos de calidad y de mayor amplitud.

Ciclos de Mejora en el Aula (2020). Experiencias de Innovación Docente de la US Esta obra se distribuye con la licencia Creative Commons 
Palabras claves: Publicidad; Relaciones Públicas; Comunicación Política; Docencia Universitaria; Experimentación Docente Universitaria.

Keywords: Advertising; Public Relations; Political Communication; University Teaching; University Teaching Experimentation.

\section{Referencias bibliográficas}

Bain, K. (2004). Lo que hacen los mejores profesores universitarios. Valencia: Universita de Valencia.

Finkel, D. (2008). Dar clase con la boca cerrada. Valencia: Universitat de Valencia.

Porlán, R. (Coord.). (2017). Enseñanza universitaria. Cómo mejorarla. Madrid: Morata.

Ciclos de Mejora en el Aula (2020). Experiencias de Innovación Docente de la US (C) $($ ) $\Theta$ Esta obra se distribuye con la licencia Creative Commons 